


\title{
Intención de generar E-WOM en Instagram derivado del encuentro con las letras monumentales turísticas
}

\author{
Ángel Hernández Morales ${ }^{1}$, Santa Fe Martínez García ${ }^{2}$ \\ y Omar Alonso Villafuerte Sánchez ${ }^{3}$ \\ IUniversidad del Noreste, angel.hernandez@une.edu.mx, Av. Miguel Hidalgo 6315, Nuevo Aeropuerto, \\ 89337, Tampico, Tamps., 8332303830 Ext. 1122. \\ ${ }^{2}$ Universidad del Noreste, smartinez@une.edu.mx, Av. Miguel Hidalgo 6315, Nuevo Aeropuerto, \\ 89337, Tampico, Tamps., 8332303830 Ext. 1122. \\ ${ }^{3}$ Universidad del Noreste, ovillafuerte@une.edu.mx, Av. Miguel Hidalgo 6315, Nuevo Aeropuerto, \\ 89337, Tampico, Tamps., 8332303830 Ext. 1122.
}

Información del artículo revisado por pares

Fecha de aceptación: junio-2021

Fecha de publicación en línea: diciembre-2021

DOI: https://doi.org/10.29105/vtga7.2-37

\section{Resumen}

Las letras monumentales turísticas (LMT) colocadas en varias ciudades de la República Mexicana, tienen distintas finalidades como las de: identificar un destino turístico, ser parte de la vista o escenografía y propiciar contenido para ser difundido a través de las redes sociales digitales (RSD). Las investigaciones respecto al uso de las RSD, como medio para producir información en Internet sobre los destinos y servicios, son de creciente interés para el sector turístico. Por lo tanto, la presente investigación tiene como objetivo principal: analizar los factores principales que inciden en la intención de generar publicidad de boca en boca en medios electrónicos (E-WOM) en RSD derivado del encuentro con las LMT. La presente investigación es cuantitativa, descriptiva y transaccional. Se aplicó una encuesta autoadministrada, con reactivos en escala de Likert de 10 niveles, de modo aleatorio y en línea a distintas universidades en territorio mexicano durante el mes de Enero del 2020. Se realizó un Análisis

\begin{abstract}
The tourist monumental letters (TML) placed in different cities of the Mexican Republic, have different purposes such as: identifying a tourist destination, being part of the view or scenery and promoting content to be disseminated through digital social networks (DSN). Research on the use of DSN as a means of producing information on the Internet about destinations and services is of growing interest to the tourism sector. Therefore, the main objective of this research is: to analyze the main factors that influence the intention to generate word of mouth advertising in electronic media (EWOM) in DSN derived from the encounter with the LMT. The present investigation is quantitative, descriptive and transactional. A self-administered survey was applied, with 10-level Likert scale reagents, randomly and online to different universities in Mexican territory during the month of January 2020. A Factor Analysis (FA) was carried out with VARIMAX rotation for the extraction of the components with the greatest significant
\end{abstract}


Factorial (AF) con rotación VARIMAX para la extracción de los componentes con mayor carga significativa. Los sujetos de estudio fueron millennials quienes manifestaron ser usuarios de Instagram y haber compartido contenido de las LMT en su perfil. Se obtuvieron 112 observaciones validadas. Finalmente, se concluye que las LMT son un elemento visual que influye en la intención de generar E-WOM en Instagram; ya que el turista es influido por este elemento visual del destino para compartir su experiencia, proporcionar opiniones y publicar imágenes de su actividad turística.

Palabras clave: letras monumentales turísticas (LMT), E-WOM, redes sociales digitales (RSD), millennials, Instagram.

\section{INTRODUCCIÓN}

A nivel gubernamental, el sector turístico en México ha sido favorecido con múltiples estrategias para la promoción y difusión de los destinos turísticos. Una de estas estrategias es la implementación de un elemento visual conocido como las letras monumentales turísticas (LMT) colocadas en varias ciudades de la República Mexicana, las cuales identifican los nombres de las ciudades y en ocasiones, logran exponer aspectos simbólicos y distintivos de los destinos. Las distintas finalidades de las LMT son: identificar un destino turístico por medio de un diseño artístico que funge como ícono distintivo del lugar; asimismo, se utilizan como parte de la vista o escenografía de los espacios públicos (Ramirez, 2018); propiciar información y contenido para ser difundido a través de las plataformas que ofrece el Internet, como las redes sociales, con la finalidad de posicionar a los diversos destinos turísticos (Pulido, 2016). Por tal motivo, este documento considera de interés particular analizar una de las finalidades de las LMT, el cual es el uso de estas como elemento motivador en la producción de contenido digital de redes sociales digitales (RSD) de los turistas. load. The study subjects were millennials who reported being Instagram users and having shared TML content on their profile. Finally, 112 validated observations were obtained. Finally, it is concluded that the TML are a visual element that influences the intention to generate E-WOM in the DSN; since the tourist is influenced by this visual element of the destination to share their experience, provide opinions and publish images of their tourist activity.

Keywords: Tourist monumental letters (TML), E-WOM, digital social networks (DSN), millenials, Instagram.

JEL: M310, M370 y M390.

\subsection{Las redes sociales digitales en el turismo}

La industria turística ha sido susceptible a los cambios provocados por la incursión de las RSD. En una investigación elaborada por Miranda y Cruz (2016) se expone que las RSD, dentro del turismo, son consideradas como medios y formas revolucionarias para crear lazos, como las llamadas comunidades virtuales o fandoms; y de utilidad si se implementan como estrategias para buscar nuevas oportunidades de negocio e igualmente incidir en la conducta de los usuarios. Aunado a esto, los autores manifiestan que con la expansión de los teléfonos inteligentes, cualquier persona tiene la posibilidad de estar conectada a una red social desde diferentes puntos del planeta... una red social puede convertirse en la mejor herramienta tanto para expandir una empresa al extranjero como para asentar un comercio en un país extranjero en el que ya se encuentra presente. (Miranda \& Cruz, 2016, pág. 2)

Por otra parte, Yoo et al. (2011) señalan que las RSD son efectivas para que los viajeros obtengan un conocimiento más completo sobre un producto o destino turístico comparado con otras fuentes de información formales como las producidas por las propias marcas o empresas de servicios turísticos. Además, el uso de las RSD es significativo en la fase de investigación del proceso de 
planificación de viajes; debido a que los consumidores, además de recopilar información de viajes de amigos y familiares que se encuentran dentro de su red social, también pueden obtener información más amplia de usuarios de Internet de todo el mundo; además, las RSD influyen en las decisiones de las actividades o servicios que se consumen durante el viaje, como el servicio de hospedaje, alimentación, actividades y visita de atractivos turísticos, etc. (Leung et al., 2013).

Uno de los tantos comportamientos que se han desarrollado en las RSD es la de producir recomendaciones de los destinos y servicios turísticos. A este comportamiento se le conoce como publicidad de boca en boca por medios electrónicos, llamada electronic word of mouth (E-WOM). Litvin et al., (2008) define al E-WOM como aquellas comunicaciones informales entre los usuarios de Internet, donde el contenido del mensaje es relacionado con el uso o las características de bienes y servicios particulares o de los proveedores de estos. Algunas formas usuales del E-WOM son recomendaciones, calificaciones u opiniones en línea, los cuales han ganado importancia en los medios electrónicos ente los consumidores (Chevalier \& Mayzlin, 2006).

\subsection{Problema de investigación}

Las investigaciones respecto al uso de las $\mathrm{RSD}$, como medio para producir información en Internet sobre los destinos y servicios, son de creciente interés para el sector turístico. Este argumento concuerda con lo expuesto por Luo \& Zhong (2015), quienes declaran que las RSD son un tema de investigación popular en el campo del turismo; así también con Munuar \& Jacobsen (2014), quienes expresan la creciente relevancia de las RSD en las actividades y estrategias de los destinos y empresas turísticas. Baksi (2016) expone que las RSD ofrecen una alternativa para producir una vinculación con el destino, la cual es el resultado de los valores físicos y los valores emocionales, en una expresión cognitiva híbrida utilizando imágenes y texto.

Derivado del interés del uso de las RSD y los contenidos tipo E-WOM, se ha encontrado que, existen dos tendencias de estudio a nivel individuo. Por un lado, aquellas que exploran las motivaciones de los usuarios para generar contenido (Huang et al., 2010; Munar \& Jacobsen, 2014; Williams et al., 2015); y por el otro lado, los efectos que producen las RSD y el E-WOM en la audiencia o consumidores potenciales (Yoo \& Gretzel, 2011; Leung et al., 2013; Setiawan et al., 2014). Respecto a las aportaciones pertenecientes a la línea de los efectos producidos en la audiencia o consumidores potenciales, se observa que éstas declaran distintos objetivos de investigación, por ejemplo: examinar la relación causal entre el contenido de las RSD y la imagen del destino, la satisfacción y lealtad (Setiawan et al., 2014); analizar y revisar las aportaciones científicas en un estudio longitudinal sobre los contenidos y efectos de las RSD en el turismo (Leung et al., 2013); proporcionar información práctica para el marketing en plataformas de medios generados por el consumidor y la gestión de foros de consumidores en línea (Yoo, Gretzel, \& Zach, 2011). Empero, ninguno de los objetivos de los artículos científicos mencionados se enfoca en analizar como algún icono representativo, que para el caso de la presente investigación son las LMT, puede incidir en la intención de producir EWOM.

Por lo tanto, la presente investigación tiene como objetivo principal: analizar los factores principales que inciden en la intención de generar E-WOM en RSD derivado del encuentro con las LMT.

\section{MARCO TEÓRICO DEL E-WOM}

El E-WOM puede ser considerado como un mensaje confiable, de una fuente informal, la cual solo pretende compartir una experiencia respecto a un producto o servicio. En términos de turismo, una recomendación del destino, de la atracción o de la empresa de servicios vinculados a esta actividad que influye en las expectativas de la audiencia o consumidor potencial. Este argumento concuerda con los hallazgos principales de la investigación de Narangajavana et al., (2017), quienes manifiestan que cuando los turistas reciben contenidos generados por el usuario y 
relacionados con destinos turísticos, crearán expectativas sobre el destino al depositar su confianza en los contenidos recibidos. Setiawan et al., (2014) indican que el E-WOM tiene un efecto directo significativo en la imagen de destino; refiriéndose al constructo de imagen del destino como un conjunto de conocimientos e impresiones de los viajeros locales, así como la evaluación de la atracción. Por lo que, el E-WOM es un proceso comunicativo, donde el receptor llega a producir una exceptiva con base en la información que recibe de la percepción general que tiene el emisor.

En las RSD el E-WOM mantiene la confianza generada por los intercambios interpersonales, subsana la falta de contacto personal y la débil credibilidad, a consecuencia de algunos factores como la popularidad de estas plataformas, los perfiles personales visibles $\mathrm{y}$ las redes sociales integradas; por lo que, se considera que las RDS heredan la naturaleza interpersonal de la publicidad de boca en boca, llamada WOM por sus siglas en inglés (Yen \& Tang, 2015).

\subsection{El E-WOM en Instagram}

En el contexto del turismo, el propósito de la fotografía en plataformas online es crear o influir en la percepción del destino turístico. Las fotografías creadas de los usuarios en RSD adquieren el roll de símbolo de la realidad y de la experiencia del viaje; además, son comentadas, compartidas y susceptibles a integrar herramientas propias de las RSD como la etiqueta o mención, así como adherir la ubicación geográfica. Leung et al., (2013) establece que, el contenido generado por el usuario en las RSD, que incluye la capacidad de compartir fotos, además de videos y comentarios con otros lectores, tiene la capacidad para impulsar a las empresas de turismo y la hospitalidad, captar clientes potenciales, aumentar su presencia en línea y, por lo tanto, producir una mayor cantidad de ingresos.

Por su naturaleza gráfica, Instagram se ha caracterizado como una plataforma significativa para la promoción de la oferta turística a través de la fotografía del destino. El contenido visual puede ser creado como estrategia por parte de alguna marca, empresa o destino; así como generado de modo orgánico por parte de los usuarios. En Instagram, según Fatanti \& Suyadnya, las fotografías son el contenido principal y coadyuvan a capturar la realidad del turista que trata de compartir su realidad con otros; también, el uso de esta plataforma mediante un teléfono inteligente propicia que las capturas se vuelvan agradables y fáciles (2015).

Por otra parte, Gosal, et al. (2020) declara que Instagram, además de ser una RSD en donde se comparten fotos y videos que pueden generar intenciones y decisiones de viaje, se ha convertido en el foco principal de los especialistas en marketing para hacer marketing. Este interés se debe a la facilidad para generar contenido visual en Instagram y hace que esta RSD sea una "referencia para que las personas busquen una descripción general de un lugar o ubicación turística en un área" (Priadana \& Habibi, 2019, pág. 6).

Además, el contenido visual en Instagram puede ser cargado con un texto de descripción que comunica la intención del usuario detrás de la publicación de la imagen y que contiene una carga emocional (Fiallos, et al., 2018). Por los atributos mencionados, las imágenes del turista en Instagram pueden ser consideradas como contenido tipo EWOM. Aunado a esto, en Instagram, como en la mayoría de las RSD, se propicia un E-WOM interactivo; donde los usuarios son creadores de opiniones, buscadores y transmisores al mismo tiempo (Chu \& Kim, 2011).

\subsection{Dimensiones del E-WOM}

Alhidari et al., (2015) ha considerado a las RSD como un medio de publicidad y una plataforma de gran popularidad y auge en los jóvenes actuales; por tal razón, las empresas están invirtiendo recursos sustanciales para incrementar el E-WOM. El estudio de este autor buscó identificar antecedentes claves a nivel personal como la participación, la autosuficiencia y la toma de riesgos para incidir en la intención de producir E-WOM y finalmente, como este comportamiento de publicidad de boca en boca incide en la intención de compra. Los datos obtenidos mediante una encuesta autoadministrada y 
analizada por medio de Modelos de Ecuaciones Estructurales (MES) indican que la participación y la toma de riesgos inciden en la intención de generar E-WOM; sin embargo, con la autosuficiencia se careció de una relación significativa. Finalmente, el autor indica que las dimensiones que influyen en propiciar E-WOM son la necesidad de: compartir experiencias, proporcionar opiniones, publicar contenido gráfico y considerar a las RSD como una fuente de información importante.

\subsection{Hipótesis de la investigación}

Derivado de la revisión teórica, la presente investigación propone las siguientes hipótesis:

H1: Compartir experiencias de las LMT incide en la intención de generar E-WOM en Instagram.

H2: Proporcionar opiniones de las LMT incide en la intención de generar E-WOM en Instagram.

H3: Publicar imágenes de las LMT incide en la intención de generar E-WOM en Instagram. H4: Considerar importante el contenido de las LMT en Instagram incide en la intención de generar E-WOM.

Figura 1. Modelo hipotético de la investigación.

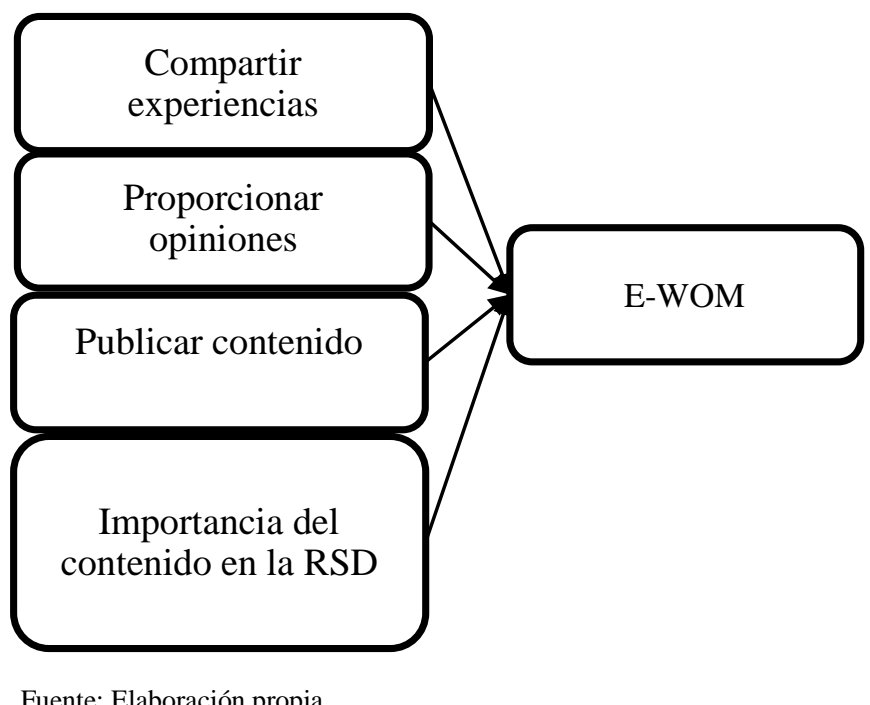

Fuente: Elaboración propia

\section{MÉTODO}

La presente investigación es cuantitativa, descriptiva y de corte transaccional. Se diseñó una encuesta auto administrada para que fuera distribuida de modo aleatorio, en línea, a distintas instituciones de educación de nivel universitario en territorio mexicano, durante el mes de Enero del 2020. Dicho instrumento contó con reactivos de filtro, útiles para asegurar el perfil de los encuestados, así como con reactivos medidos en escala de Likert de 10 niveles precedidos por un enunciado declarativo. Cabe mencionar que, el instrumento fue una adaptación de la encuesta aplicada en el estudio realizado por Alhidari, Iyer y Paswan (2015).
Las pruebas y análisis estadísticos que se utilizaron en esta investigación son las medidas de tendencia centralizada para los estadísticos descriptivos; el Alpha de Cronbach para la fiabilidad del instrumento; la prueba de KMO y la esfericidad de Barlett para asegurar el empleo del modelo estadístico seleccionado; el Análisis Factorial (AF) con rotación VARIMAX para la extracción de los componentes con mayor carga significativa.

\subsection{Delimitaciones del estudio}

En cuestión espacial, esta investigación se enfoca en las LMT instaladas en destinos turísticos del territorio mexicano, para considerar actividades exclusivamente del tipo catalogado como turismo interno. 
Además, esta investigación se centró en la RSD llamada Instagram. Esto atiende principalmente a la existencia de un gran número de RSD, con diferentes características y herramientas; además, la naturaleza de cada RSD influye en el comportamiento online de los usuarios. Por lo que, de haber realizado el estudio considerando simultáneamente distintas RSD, se pudo ocasionar una heterocedasticidad al tener diferentes comportamientos en distintas plataformas.

En cuestión demográfica, los sujetos de estudio son aquellos denominados como millennials, nacidos entre los años de 1980 al 2000; este corte generacional es mencionado por varios investigadores al igual por Cuesta et al. (2008). Los millennials son un tipo de usuario que manifiestan una mayor tendencia o frecuencia de uso de plataformas catalogadas como RSD y son conocidos por ser nativos digitales (Hershatter \& Epstein, 2010). Este tipo de usuario se siente cómodo con los ambientes colaborativos y logra una comunicación mediada por un dispositivo electrónico para obtener intercambios más ricos y extensos a diferencia de una situación presencial cara a cara (Wankel, 2009); desarrollan eficacia en la multitarea, a la respuesta de estimulación visual, filtrado de información, dado que la tecnología para ellos es como un sexto sentido, una forma de conocer e interactuar con el mundo (Hershatter \& Epstein, 2010). Además, los sujetos considerados para la muestra debieron haber realizado viajes o desplazamientos turísticos entre enero del 2017 y diciembre del 2019; haber declarado ser usuarios de Instagram, asî como reconocer la existencia de las LMT y haber generado contenido visual sobre ellas en su perfil de Instagram durante o después de su viaje.

\section{RESULTADOS}

Después del piloteo y mejoras del instrumento, este se dispersó y se obtuvieron un total de 494 observaciones, 249 resultaron ser mujeres y 115 hombres, todos ellos millennials, usuarios de Instagram y reconocen la presencia de las LMT. Además, 237 encuestados manifestaron haber capturado su visita a las LMT en una fotografía durante su viaje; de estos, solo 112 expresan haber publicado la foto en su perfil de Instagram. Estas 112 observaciones fueron consideradas como las válidas para continuar con el AF al cumplir con las delimitaciones demográficas del estudio. Los resultados descriptivos de la encuesta se pueden apreciar en la Tabla 1 a continuación.

Tabla 1 Resultados de la encuesta

\begin{tabular}{lcc}
\hline \multicolumn{1}{c}{ Características de las observaciones } & Totales & $\%$ \\
\hline Encuestados & 494 & 100 \\
Reconocen la presencia de la LMT & 494 & 100 \\
Capturó en una fotografía su visita a las & 237 & 47.97 \\
LMT & $\mathbf{1 1 2}$ & $\mathbf{2 2 . 6 7}$ \\
Publicó la fotografía de las LMT en & & \\
Instagram
\end{tabular}

Fuente: Elaboración propia.

Posteriormente, los datos fueron procesados mediante el software SPSS y se obtuvieron los siguientes resultados: 1) coeficiente del Alpha de Cronbach de .898, el cual indicó fiabilidad en el instrumento, ya que superó el límite inferior de .70 para mostrar consistencia (Rositas, 2014); 2) coeficiente de la prueba de KMO de .880; y 3) la prueba de esfericidad de Barlett de .000; estas dos últimas pruebas indicaron que el uso del AF, para el tratado de los datos, era conveniente.

Los resultados del AF mostraron que, con tres componentes, cantidad inferior a la propuesta en el modelo hipotético, se puede explicar el $70.84 \%$ de la varianza, tal y como se muestra en la Tabla 2. A continuación, en la Tabla 3, se observan las cargas factoriales de cada variable, donde resalta la $\mathrm{H} 4$ : Considerar importante el contenido de las LMT en Instagram, ya que careció de una carga significativa en la intención de generar EWOM. El resto de las variables obtuvieron cargas consideradas como significativas, siendo H1: Compartir experiencias de las LMT incide en la intención de generar EWOM en Instagram.

Tabla 2 Varianza explicada

\begin{tabular}{ccccccc}
\hline & \multicolumn{3}{c}{ Varianza } & \multicolumn{4}{c}{$\begin{array}{c}\text { Sumas de rotaciones de cargas } \\
\text { al cuadrado }\end{array}$} \\
& Total & $\%$ & $+\%$ & Total & $\%$ & $+\%$ \\
\hline 1 & 5.501 & 50.01 & 50.01 & 2.742 & 24.924 & 24.924 \\
2 & 1.302 & 11.832 & 61.842 & 2.703 & 24.571 & 49.494 \\
3 & 0.99 & 9.004 & 70.846 & 2.349 & 21.352 & $\mathbf{7 0 . 8 4 6 *}$ \\
4 & 0.74 & 6.727 & 77.573 & & &
\end{tabular}






Fuente: Elaboración propia.

Nota:

*Varianza explicada.

$+\%$ Porcentaje acumulado de varianza.

Tabla 3. Cargas factoriales.

\begin{tabular}{lccc}
\hline \multicolumn{1}{c}{ Hipótesis } & Cargas & Cargas & Cargas \\
\hline H1 Compartir experiencias & .252 & .203 & $\mathbf{. 8 1 9}$ \\
H2 Proporcionar opiniones & .392 & .161 & $\mathbf{. 7 5 7}$ \\
H3 Publicar imágenes & .115 & .392 & $\mathbf{. 7 1 3}$ \\
$\begin{array}{l}\text { H4 Considerar importante el } \\
\quad \text { contenido de Instagram }\end{array}$ & .588 & .114 & .508 \\
\hline
\end{tabular}

Fuente: Elaboración propia.

\section{CONCLUSIONES}

\section{RECOMENDACIONES}

Las LMT representan aspectos simbólicos de la cultura del lugar en donde se ubican. Asimismo, son un elemento visual que provoca interés y atracción. Este argumento se sustenta en el resultado de las encuestas, en donde el total de los millennials encuestados declara reconocerlas y el $47.97 \%$ de ellos, manifestó haber capturado en imagen su visita a las LMT. Con respecto al análisis de los datos de aquellas observaciones en donde se declaró que la imagen capturada se publicó en Instagram, el estudio concluye que las LMT son un elemento visual que influye en la intención de generar E-WOM en las RSD, específicamente en Instagram; ya que el turista es influido por este elemento visual del destino para compartir su experiencia, proporcionar opiniones y publicar imágenes de su actividad turística. Por lo tanto, se cumple una de las finalidades de las LMT, la cual es la de generar tráfico online del destino.

En conclusión, las LMT, ubicadas en los destinos, influyen sobre la intención de generar E-WOM en Instagram, ya que derivado del encuentro con ellas el usuario tiende a compartir sus experiencias, propiciar opiniones y publicar las imágenes. Por otro lado, la importancia de Instagram, como fuente de información, carece de incidencia significativa en la intención de generar EWOM. En la siguiente Tabla 4 se exponen las conclusiones de las hipótesis del modelo propuesto.
Tabla 4. Conclusión del modelo hipotético.

\begin{tabular}{|c|c|c|}
\hline Hipótesis & Aceptada & Rechazada \\
\hline $\begin{array}{l}\text { H1: Compartir experiencias de } \\
\text { las LMT incide en la intención } \\
\text { de generar E-WOM en } \\
\text { Instagram. }\end{array}$ & $X$ & \\
\hline $\begin{array}{l}\text { H2: Proporcionar opiniones de } \\
\text { las LMT incide en la intención } \\
\text { de generar E-WOM en } \\
\text { Instagram. } \\
\text { H3: Publicar imágenes de las } \\
\text { LMT incide en la intención de } \\
\text { generar E-WOM en Instagram. } \\
\text { H4: Considerar importante el } \\
\text { contenido de las LMT en } \\
\text { Instagram incide en la intención } \\
\text { de generar E-WOM. }\end{array}$ & $X$ & $X$ \\
\hline
\end{tabular}

Finalmente, el uso de las LMT en los destinos de México es una estrategia gubernamental significativa que logran influir en la producción de tráfico de información en las RSD. Por tal motivo, es necesario y recomendable que el sector público y privado realicen esfuerzos similares a este tipo de estrategias para generar íconos visuales representativos de los destinos y que logren motivar a los turistas a la adopción de un papel o rol tipo prosumer.

Entiéndase como prosumer al anglicismo producido por la combinación de las palabras producto y consumidor, definiéndose como aquel "usuario que crea y produce contenidos en la red" (Herrero, Ramos, \& Nó, 2016, pág. 1305) y que después o durante su proceso de consumo comparten sus experiencias y son influencia para otros potenciales consumidores (Flores \& Calderón, 2020).

Para efectos de futuras investigaciones en la línea del presente estudio, se recomienda que: 1) Se analicen distintas RSD y se comparen con los hallazgos de la presente, debido a que existe la posibilidad de haber creado un sesgo en la investigación ocasionado por el enfoque de análisis exclusivo del contenido producido en Instagram; lo cual, pudo influir en que la $\mathrm{H} 4$ fuera rechazada, ya que en el estudio del cual se consideró, se expone como variable las RSD y no una red social específica. 2) Incluir variables espaciales como el tipo de destino, el 
tipo de turismo que se realiza y la ubicación geográfica o estratégica de las LMT. 3) Incluir variables demográficas como el género del turista y el nivel socio económico. 4) Evaluar la incorporación de variables conductuales como el interés o motivo del viaje y el apego a las RSD. 5) Ampliar el modelo hipotético buscando analizar relaciones entre el E-WOM y otros comportamientos de consumo como la intención de visita, la imagen del destino o la creación de expectativas. 


\section{REFERENCIAS}

Alhidari, A., Iyer, P., \& Paswan, A. (2015). Personal level antecedents of eWOM and purchase intention, on social networking sites. Journal of Customer Behaviour, 14(2), [107-125].

Baksi, A. (2016). Destination bonding: Hybrid cognition using Instagram. Management Science Letters, 6(1), [31-46].

Chevalier, J. A., \& Mayzlin, D. (2006). The effect of word of mouth on sales: Online book reviews. Journal of marketing research, 43(3), [345-354]. Obtenido de https://www.nber.org/papers/w10148.pdf

Chu, S. C., \& Kim, Y. (2011). Determinants of consumer engagement in electronic word-of-mouth (eWOM) in social networking sites. International journal of Advertising, 30(1), [47-75].

Cuesta, E. M., Ibáñez, E., Tagliabue, R., \& Zangaro, M. B. (2008). El impacto de la generación millennial en la Universidad: un estudio exploratorio. Trabajo presentado en XV Jornadas de Investigación y Cuarto Encuentro de Investigadores en Psicología del Mercosur (págs. 200202). Bueno Aires: Facultad de Psicología - Universidad de Buenos Aires. Obtenido de https://www.aacademica.org/000-032/288

Fatanti, M. N., \& Suyadnya, I. W. ( 2015). Beyond User Gaze: How Instagram Creates Tourism Destination. Trabajo presentado en 2nd Global Conference on Business and Social Science2015, GCBSS-2015 (págs. 1089 - 1095 ). Bali, Indonesia: Procedia - Social and Behavioral Sciences.

Fiallos, A., Jimenes, K., Fiallos, C., \& Figueroa, S. (2018). Detecting topics and locations on Instagram photos. En: 2018 International Conference on eDemocracy \& eGovernment (ICEDEG) [246-250]. Ambato, Ecuador. Ed: IEEE.

Flores, R. \& Calderón, J. (2020). Perscetivas del prosumer: caractetísticas de decisión en el sector hotelero. Journal Business Science, [54-62].

Gosal, J., Andajani, E. \& Rahayu, S. (2020). The effect of e-WOM on travel intention, travel decision, city image, and attitude to visit a tourism city. En: 17th International Symposium on Management (INSYMA 2020) [261-265]. Ba Ria Vung Tau University, Vietnam. Ed: Atlantis Press.

Hammock, G., \& Richardson, D. S. (2011). Love attitudes and relationship experience. The Journal of Social Psychology, 151(1), [608-624].

Herrero, P., Ramos, M. \& Nó, J. (2016). Los menores como usuarios creadores en la era digital: del prosumer al creador colaborativo. Revista latina de comunicación social, [1301-1322]

Hershatter, A., \& Epstein, M. (2010). Millennials and the world of work: An organization and management perspective. Journal of business and psychology, 25(2), 211-223.

Huang, Basu, \& Hsu. (2010). Exploring motivations of travel knowledge sharing on social network sites: an empirical investigation of US college students. Journal of hospitality marketing \& management, 19(7), [717-734].

Leung, D., Law, R., Van Hoof, H., \& Buhalis, D. (2013). Social media in tourism and hospitality: A literature review. Journal of travel \& tourism marketing, 30(1-2), [3-22].

Litvin, S. W., Goldsmith, R. E., \& Pan, B. (2008). Electronic word-of-mouth in hospitality and tourism management. Tourism management, 29(3), [458-468].

Luo, Q., \& Zhong, D. (2015). Using social network analysis to explain communication characteristics of travel-related electronic word-of-mouth on social networking sites. Tourism Management, 46, [274-282].

Mauri, A. G., \& Minazzi, R. (2013). Web reviews influence on expectations and purchasing intentions of hotel potential customers. International Journal of Hospitality Management, 34, [99-107].

Miranda, A., \& Cruz, I. (2016). Redes sociales: herramienta de marketing internacional en el sector hotelero. $\quad E l \quad$ periplo sustentable. Disponible en https://documentcloud.adobe.com/link/track?uri=urn\%3Aaaid\%3Ascds\%3AUS\%3A84a1d b10-b6c9-4fa2-a68e-80a858625efc. Consultado el 03/05/2019. 
Molinillo, S., Liébana, F., Anaya, R., \& Buhalis, D. (2018). DMO online platforms: Image and intention to visit. Tourism management, 65, [116-130].

Munar, A. M., \& Jacobsen, J. (2014). Motivations for sharing tourism experiences through social media. Tourism management, [46-54].

Narangajavana, Y., Fiol, L. J., Tena, M. Á., Artola, R. M., \& García, J. S. (2017). The influence of social media in creating expectations. An empirical study for a tourist destination. Annals of Tourism Research, 65, [60-70].

Priadana, A. \& Habibi, M. (2019). Face detection using haar cascades to filter selfie face image on instagram. En: 2019 International Conference of Artificial Intelligence and Information Technology (ICAIIT) [pp. 6-9]. Yogyakarta, Indonesia. Ed: IEE.

Pulido, C. G. (16 de Mayo de 2016). Mexico a la carta. Obtenido de http://mexicoalacarta.com.mx/uncategorized/entrega-secretaria-de-turismo-letrasmonumentales-al-municipio-del-marques/

Ramirez, M. (17 de Septiembre de 2018). NOTIHoteles. Obtenido de http://notihoteles.com/2018/09/17/las-letras-monumentales-de-mexico-para-el-mundogaleria/

Rositas, J. (2014). Los tamaños de las muestras en encuestas de las ciencias sociales y su repercusión en la generación del conocimiento. Innovaciones de negocios, 11(22), [235-268].

Setiawan, P. Y., Torena, E., \& Armanu, N. (2014). The Effect of e-WOM on Destination Image, Satisfaction and Loyalty. International Journal of Business and Management Invention, [2229].

Wankel, C. (2009). Management education using social media. Organization Management Journal, 6(4), [251-262].

Williams, N. L., Inversini, A., Buhalis, D., \& Ferdinand, N. (2015). Community crosstalk: an exploratory analysis of destination and festival eWOM on Twitter. Journal of Marketing Management, 31(9-10), [1113-1140].

Yen, C.-L., \& Tang, C.-H. (2015). Hotel attribute performance, eWOM motivations, and media choice. International Journal of Hospitality Management, [79-88].

Yoo, K.-H., Gretzel, U., \& Zach, F. (2011). Travel Opinion Leaders and Seekers. En R. Law, M. Fuchs, \& F. Ricci, Information and Communication Technologies in Tourism. Publicado en Proceedings of the International Conference in Innsbruck [págs. 525-535]. Austria: Springer Vienna. 


\section{APÉNDICE}

A continuación, se muestra el instrumento utilizado en la investigación.

Instrumento para analizar la relación entre el contenido producido por millennials en Instagram sobre las letras monumentales turísticas y la intención de generar E-WOM.

1. ¿Sexo? ${ }^{\bullet}$ Mujer ${ }^{\top}$ Hombre

2. Lugar de residencia.

Ciudad/Localidad $* \longdiv { }$ Estado/Provincia

3. ¿En qué año naciste? (Ingresa los 4 dígitos de tu año de nacimiento; por ejemplo, 1976)

4. ¿Eres usuario de Instagram? Sí $^{\bullet}$ No

5. ¿Realizaste algún viaje, con un más de un día de estancia, en algún destino turístico de la

República Mexicana entre enero 2017 y diciembre 2019? ${ }^{\complement}$ Sí No

6. ¿Has visto algunas de las letras monumentales turísticas instaladas en varias ciudades y destinos de la República Mexicana?

Un ejemplo de estas se muestra a continuación (inserción de imágenes de LMT)

Sí he visto alguna ${ }^{\bullet}$ NO he visto ninguna

7. ¿Te tomaste alguna foto con las letras monumentales turísticas como escenario en un destino turístico de México? Sí No

8. ¿Publicaste en Instagram alguna foto con las letras monumentales turísticas como escenario en un destino turístico de México? ${ }^{\bullet}$ Sí $^{C}$ No

Instrucciones: Lee el supuesto marcado con un \# que está antes del listado de preguntas; después marque la opción de respuesta que refleje con mayor grado su percepción; considere que 1 es el nivel más bajo (totalmente desacuerdo) y el 10 es el nivel más alto (totalmente de acuerdo).

\section{\#Cuando me he llegado a tomar fotos con las letras monumentales turísticas} en algún destino yo...

9. Publico las fotos en Instagram.

$\begin{array}{lllllllll}12 & 3 & 4 & 5 & 6 & 7 & 8 & 9 & 10\end{array}$

Totalmente Totalmente

desacuerdo de acuerdo

10. Siempre comparto mis experiencias con otros miembros en Instagram.

Totalmente

$\begin{array}{lllllllll}12 & 3 & 4 & 5 & 6 & 7 & 8 & 9 & 10\end{array}$

desacuerdo

11. Siempre proporciono mis opiniones con otros miembros en Instagram

$\begin{array}{llllllllll}1 & 2 & 3 & 4 & 5 & 6 & 7 & 8 & 9 & 10\end{array}$

Totalmente

desacuerdo

12. Las publico en Instagram porque es una fuente importante de información.

Totalmente de acuerdo

Totalmente de acuerdo

Totalmente desacuerdo 